\title{
GPPS-CH-2020-0104
}

\section{Large-Eddy Simulations of Rim Seal Flow in a One-Stage Axial Turbine}

\author{
Thomas Hösgen \\ Institute of Aerodynamics, \\ RWTH Aachen University \\ t.hoesgen@aia.rwth-aachen.de \\ Aachen, Germany
}

\author{
Matthias Meinke \\ Institute of Aerodynamics, \\ RWTH Aachen University \\ m.meinke@aia.rwth-aachen.de \\ Aachen, Germany
}

\author{
Wolfgang Schröder \\ Institute of Aerodynamics, \\ RWTH Aachen University \\ office@aia.rwth-aachen.de \\ Aachen, Germany
}

\begin{abstract}
The flow field in a one-stage axial flow turbine with 30 stator and 62 rotor blades including the wheel space is investigated by large-eddy simulation (LES). The Navier-Stokes equations are solved using a massively parallel finite-volume solver based on a Cartesian mesh with immersed boundaries. The strict conservation of mass, momentum, and energy is ensured by an efficient cut-cell/level-set ansatz, where a separate level-set solver describes the motion of the rotor. Both solvers use individual subsets of a shared Cartesian mesh, which they can adapt independently. The focus of the analysis is on the flow field inside the rotor stator cavity between the stator and rotor disks. Two cooling gas mass flow rates are investigated for the same rim seal geometry. First, the time averaged flow field for both simulations is compared, followed by a detailed investigation of the unsteady flow field. The results for the cooling effectiveness are compared to experimental data. Both cases show good agreement with experimental data. It is shown that for the lower cooling gas mass flux several of the wheel space's acoustic waves are excited. This is not observed for the higher cooling gas mass flux. The excited waves lead to stable, i.e., bounded, fluctuations inside the wheel space and result in a significantly higher hot gas ingestion.
\end{abstract}

\section{INTRODUCTION}

The thermal efficiency of gas turbines strongly depends on the turbine inlet temperature. Increasing the inlet gas temperature for an improved engine performance results in several new challenges regarding the cooling of the machine. For instance, entering of hot gas from the main annulus flow into the wheel space between the stator and the rotor disks would lead to overheating and a significant reduction of the turbine lifetime. To avoid a hot gas ingress, cooling air from the turbine's secondary air system is introduced into the wheel space, on the one hand, to cool the wheel space and on the other hand, to seal the rim seal gap at the stator hub. The primary goal is to minimize this cooling gas flow since the amount of secondary air directly deteriorates the turbine efficiency. Additionally, the mixing of the exiting cold gas with the main flow and its interaction with secondary flow structures, such as the vane hub passage vortex, causes further aerodynamic losses. The later has, for example, been investigated by (Schuepbach et al. 2010) and (Schädler et al. 2017).

Due to the large impact on the engine performance, the rim seal flow has been extensively studied over the past 50 years. A recent review of the progress made is given by (Chew et al. 2019). Early studies of rim seal ingestion focused on the steady flow field inside the rotor-stator wheel space and the turbine hub region near the rotor-stator cavity. Two main driving factors of hot gas ingestion were determined (Johnson et al. 1994). The first effect is termed rotationally-induced ingress and can even occur without blading. Inside the wheel space the cooling air is radially pumped outward in the rotor disk boundary layer. This is also commonly called disk pumping effect. Further, the acceleration of the fluid by the rotor leads to a radial pressure gradient inside the wheel space. If the pressure falls below the pressure inside main flow passage, the hot gas enters the wheel space cavity. The second effect is termed externallyinduced ingress and caused by non-axisymmetric pressure variations inside the main flow passage due to interactions of the stator vanes and the potential effect of the rotor blades. When the pressure in the hub region is higher or lower than inside the wheel space ingress or egress occurs.

Later studies focused on unsteady flow phenomena inside the rim seal cavity. (Bohn et al. 2003) and (Rudzinski 2009) were among the first to report unsteady pressure fluctuations inside the rim seal of a 1.5-stage turbine test rig. The unsteady flow structures were unrelated to blade passing and had significant impact on the sealing effectiveness. These unsteady phenomena were only present at low cooling gas mass flow rates and vanished abruptly when the cooling gas mass flux was sufficiently increased. The fluctuations were later confirmed by (Jakoby et al. 2004), who conducted numerical simulations based on the unsteady ReynoldsAveraged Navier-Stokes (RANS)- approach of the same 1.5stage turbine test rig. They compared solutions of several sector models with a full $360^{\circ}$ simulation. Only the $360^{\circ}$ simulation predicted a rotating mode inside the wheel space 
moving at about $80 \%$ of the rotor speed, and which had massive influence on the hot gas ingestion. They concluded that full $360^{\circ}$ simulations are necessary to predict the hot gas ingress, since a sector model prohibits periodicities with an extent larger than the sector size. Similar findings were made by (Cao et al. 2004). Unsteady RANS simulations unveiled periodic structures inside the rim seal, which were also observed in experimental studies. Complementing the conclusion of (Jakoby et al. 2004) the number of lobes of the periodic structures depended on the size of the chosen sector model.

The importance of these unsteady phenomena was later confirmed by (Laskowski et al. 2009). The comparison of unsteady RANS simulations of a turbine model with simplified rotor blading showed significantly better agreement with experimental data than steady-state simulations. Although rotor rotation was neglected, KelvinHelmholtz type fluctuations were predicted inside the rim seal gap by the unsteady simulation. Such fluctuations were also found by (Rabs et al. 2009) in their investigation of a sector model of a 1.5 stage axial turbine without blading. Adding blades, theses instabilities were still present, however, strongly reduced. Further studies showed that the unsteady flow phenomena strongly depended on the rim seal geometry (Chilla et al. 2013), and on the amount of injected cooling gas and the rotor speed (Beard et al. 2017).

(O’Mahoney et al. 2010) performed a large-eddy simulation (LES) of a small sector model of a rim seal geometry with main annulus flow and compared it to unsteady RANS and experimental results. Significant discrepancies between LES and experiment were observed and the LES showed better agreement than the unsteady RANS solution. They concluded that increasing the LES grid resolution and increasing the size of the sector will lead to better solutions.

This study intends to increase the understanding of the inherent unsteady nature of the rim seal flow in axial flow turbines and follows the work of (Pogorelov et al. 2019). They conducted high fidelity LES of an axial flow turbine for the full $360^{\circ}$ circumference of the main flow and the wheel space such that the uncertainty of RANS turbulence modelling and the limitations of a sector model was avoided. Two rim seal geometries were considered. They found Kelvin-Helmholtz type fluctuation inside a single lip axial rim seal gap, which interacted with the main annulus flow. Adding a second sealing lip supressed these fluctuations and reduced the hot gas ingress. In this paper, new results for the double lip rim seal geometry investigated by (Pogorelov et al. 2019) are presented for a cooling gas mass flow rate reduced by $50 \%$.

The paper is organized as follows. First, the governing equations and the numerical approach are discussed. Second, the computational setup, the investigated operating conditions, and boundary conditions are presented. Afterwards, the results of the flow field inside the rotor-stator wheel space are presented and compared to the results for the higher cooling gas mass flow rate from (Pogorelov et al. 2019). Finally, some conclusions are drawn.

\section{METHODOLOGY}

\section{Governing equations}

The motion of a viscous, compressible fluid is described by the Navier-Stokes equations. For an arbitrary moving control volume $V$ with surface $A$, the conservation equations for mass, momentum, and energy read

$$
\frac{d}{d t} \int_{V(t)} \boldsymbol{Q} d V+\oint_{A(t)} \overline{\boldsymbol{H}} \cdot \boldsymbol{n} d A=0 .
$$

Here, $\boldsymbol{Q}=[\rho, \rho u, \rho \mathrm{E}, \rho \mathrm{Y}]^{T}$ is the vector of the conservative variables with the density $\rho$, the velocity vector $u$, the total energy $\rho \mathrm{E}=\rho \mathrm{e}+\rho \overrightarrow{\mathrm{u}}^{2} / 2$, and the concentration of a passive scalar Y. The variable $e$ denotes the specific internal energy. The quantity $\bar{H}$ is the flux tensor and $\boldsymbol{n}$ the outward normal vector on the surface $A$. The flux tensor is split into an inviscid part

$$
\overline{\boldsymbol{H}}^{i n v}=\left(\begin{array}{c}
\rho\left(u-u_{\partial V}\right) \\
\rho u\left(u-u_{\partial V}\right)+p \bar{I} \\
\rho E\left(u-u_{\partial V}\right)+\rho u \\
\rho Y\left(u-u_{\partial V}\right)
\end{array}\right),
$$

and a viscous contribution

$$
\overline{\boldsymbol{H}}^{v i s}=\frac{1}{R e_{0}}\left(\begin{array}{c}
0 \\
\bar{\tau} \\
\bar{\tau} u+q \\
\frac{-\rho D \nabla Y}{S c_{0}}
\end{array}\right),
$$

where $u_{\partial V}$ is the velocity of the control volume's surface, $p$ is the pressure, and the unit tensor is given by $\bar{I}$. Additionally, $D$ is the mass diffusion coefficient of the passive scalar $Y$, and $S c_{0}=1$ is the Schmidt number.

All variables are given in their non-dimensional form with respect to the variables at the state of rest denoted by " 0 ". The Reynolds number and the speed of sound are expressed by

$$
R e_{0}=\left(\rho_{0} a_{0} l_{\text {ref }}\right) / \eta_{0}
$$

and

$$
a_{0}=\sqrt{\gamma p_{0} / \rho_{0}}
$$

with the ratio of specific heats $\gamma=1.4$ and the characteristic length $l_{\text {ref }}$. For a Newtonian fluid with zero bulk viscosity, the stress tensor is written

$$
\overline{\boldsymbol{\tau}}=(2 / 3) \eta(\nabla \cdot u) \overline{\boldsymbol{I}}-\eta\left(\nabla u+(\nabla u)^{T}\right) .
$$

The dynamic viscosity is computed using Sutherland's law (Sutherland 1893)

$$
\eta(T)=(T)^{3 / 2} \frac{1+S}{T+S},
$$

where $S=111 K / T_{0}$. For constant Prandtl number $P r_{0}=0.72$ the non-dimensional vector of heat conduction according to Fourier's law is

$$
\boldsymbol{q}=-\frac{\eta}{\operatorname{Re}_{0} P r_{0}(\gamma-1)} \nabla T \text {. }
$$


The equations are closed by the ideal gas relation, which is written in non-dimensional form

$$
\gamma p=\rho T \text {. }
$$

At fixed and at moving boundaries the no-slip condition is imposed. All walls are considered adiabatic. The pressure at the solid boundaries is determined via a robin-type boundary condition derived from the momentum equation.

\section{Numerical method}

The numerical method is based on a finite-volume solver and a level-set solver, which are efficiently coupled since both solvers hold individual subsets of a shared hierarchical Cartesian mesh. The shared base grid is generated by a parallel grid generator developed by (Lintermann et al. 2014). During the solver run, the mesh is adapted according to the movement of the blade surfaces. The individual subsets of the grid are adapted separately and can differ even in regions where the domains of the solvers overlap.

The conservation equations eq.(1) are discretized by a cell-centered finite-volume method. Following the largeeddy simulation (LES) approach, the turbulent structures are divided into the resolved part, which contains the dominant, large-scale, and most energetic eddies, and the unresolved part, which is modelled by the sub-grid scale model. An implicit filter is applied, where the filter width is determined by the length of the grid cells. The sub-grid contribution is modelled by the monotone integrated LES (MILES) approach (Boris et al. 1992), in which the discretization error of the numerical scheme is used to dissipate the energy at the unresolved scales.

A low-dissipation variant of the advection upstream splitting method (AUSM) proposed by (Meinke et al. 2002) is used to calculate the inviscid flux tensor $\overline{\boldsymbol{H}}^{i n v}$ eq.(2). The primitive variables at the cell surfaces are obtained by a second-order accurate MUSCL extrapolation. The cellcentered gradients are computed by a weighted least-square method described in (Schneiders et al. 2016). The viscous flux tensor $\overline{\boldsymbol{H}}^{\text {vis }}$ eq.(3) is computed using a central-difference scheme. The gradients at the cell-surface centroids are computed with the recentering-approach proposed by (Berger and Aftosmis 2012).

The time-integration is performed by an explicit secondorder accurate 5-stage Runge-Kutta scheme (Schneiders et al. 2016). This Runge-Kutta scheme is optimized for the efficient computation of moving boundaries. The RungeKutta coefficients are chosen for maximum stability. The time step fulfills the Courant-Friedrichs-Lewy (CFL) condition

$$
\Delta T=\mathrm{CFL} \cdot \min n_{i=1,2,3} \frac{h}{\left|u_{i}\right|+a},
$$

where $h$ is the length of the smallest cell, $u_{i}$ the velocity in the direction $i$ and $a$ the speed of sound.

A strictly conservative cut-cell method is used to accurately represent the surfaces of embedded bodies, which are prescribed by a zero level-set contour obtained from the level-set solver. To prevent arbitrarily small time steps, a flux redistribution method is applied in small cut-cells. A detailed description of the cut-cell method and the flux redistribution method is given in (Schneiders et al. 2016).

A level-set approach is applied to describe the movement of embedded bodies. The level-set is a signeddistance function that divides the domain into a solid $\mathcal{G}_{\gamma}^{8}$ and fluid $\mathcal{G}_{\gamma}^{\mathfrak{f}}$ part

$$
\begin{gathered}
\mathcal{G}_{\gamma}^{f}(t)=\{x \mid \Phi(x, t)>0\} \\
\Gamma(t)=\{x \mid \Phi(x, t)=0\}, \\
\mathcal{G}_{\gamma}^{\mathcal{8}}(t)=\{x \mid \Phi(x, t) \leq 0\} .
\end{gathered}
$$

The surface of the body is given by the zero level-set contour $\Gamma$. To preserve the resolution of the initial level-set surface while moving the bodies, the kinematic motion levelset approach described by (Pogorelov et al. 2018) is used. In this method, the level-set solution at each time step is interpolated from the initial level-set field at the zeroth time step, which is reconstructed from high resolution stereolithography (STL)-data. Thus, the error in the surface representation only depends on the interpolation error and remains constant over time. This renders the method superior regarding other level-set approaches, such as the use of levelset transport equations, where the accuracy of the geometry representation can deteriorate over time.

A more general description of the solver can be found in (Lintermann et al. 2020), where more details of the solver structure are described along with performance measures.

\section{Computational setup}

The one-stage axial flow turbine shown in Figure 1 is investigated. The stator and rotor are separately illustrated for the purpose of better visualisation. The turbine stage consists of 30 stator vanes and 62 rotor blades. A double lip rim seal is used to seal the rotor-stator wheel space. The upper seal lip is mounted on the stator and the second lip is mounted on the rotor disk. The turbine has been previously investigated by, for example, (Bohn and Wolff 2003). Detailed experimental data is available in (Bohn and Wolff 2001).

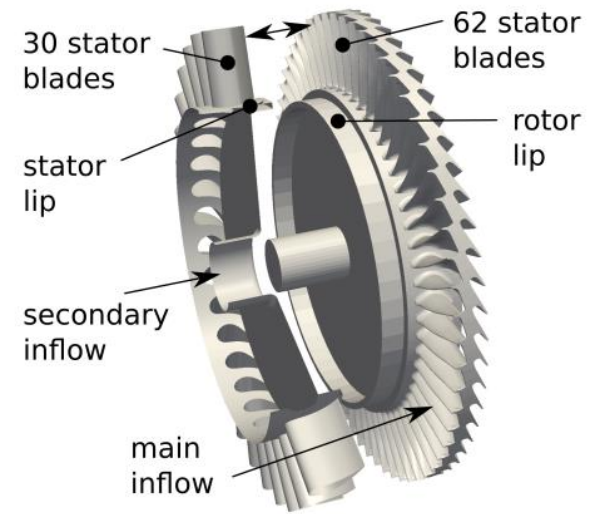

Figure 1 Cut through the one-stage axial flow turbine; rotor and stator are separated 
The full $360^{\circ}$ circumference of the main annulus flow and wheel space is resolved by an adaptive Cartesian mesh with approximately 450 million cells. The subset used by the finite-volume solver includes approximately 400 million leaf cells of the shared grid, while the level-set solver uses about 450 million leaf cells. Figure 2 shows an axial cut through the mesh. Two layers of refinement are used at the fixed and the moving boundaries. The size of the smallest regular, uncut cell is approximately $0.0017 \mathrm{R}$. A zoom of the rim seal geometry for the region marked in Figure 2 by the dashed line is displayed in Figure 3.

The operating conditions are defined by the four dimensionless quantities listed in Table 1. The subscript 1 indicates the flow state $1.5 \mathrm{~mm}$ downstream of the stator blades and cg denotes the conditions inside the cooling gas inlet. Two LES are conducted. Both LES share the same main flow operating condition $\left(\mathrm{Re}_{1}, \mathrm{M}_{1}\right)$ and rotor speed $\mathrm{Re}_{\mathrm{u}}$. They differ in the dimensionless cooling gas mass flux $\mathrm{cw}$, which is reduced by $50 \%$ in $\mathrm{CW} 1 \mathrm{~K}$ compared to $\mathrm{CW} 2 \mathrm{~K}$.

At the main and secondary inflow boundaries the density and the three velocity components are prescribed. Additionally, the velocity at the main inflow is superimposed by a synthetic turbulence with 5\% turbulence intensity (Batten et al. 2004). At the outflow boundary, the static pressure is fixed. To reduce numerical wave reflections sponge layers are used at all in- and outflow boundaries. At the fixed and the moving walls, an adiabatic no-slip condition is prescribed.

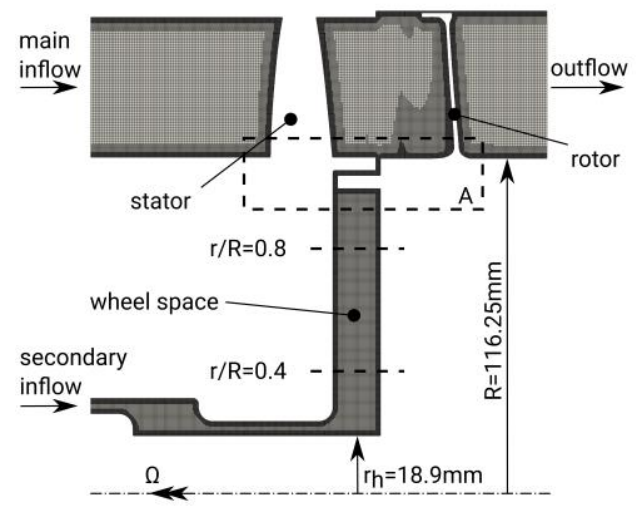

Figure 2 Axial cut through the computational mesh

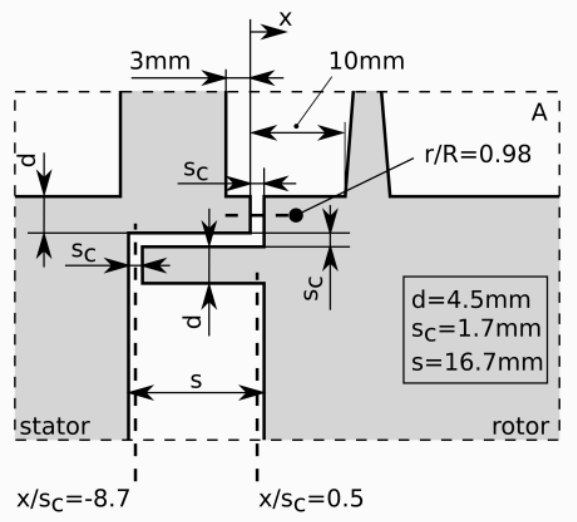

Figure 3 Schematic view of the rim seal geometry
Table 1 Operating conditions

\begin{tabular}{|l|c|c|}
\hline & CW1K & CW2K \\
\hline $\mathrm{Re}_{1}=\frac{\rho_{1} \mathrm{u}_{\mathrm{ax}, 1} \mathrm{R}}{\eta_{1}}$ & $0.8 * 10^{6}$ & $0.8 * 10^{6}$ \\
\hline $\mathrm{M}_{1}=\frac{\mathrm{u}_{\mathrm{ax}, 1}}{\sqrt{\gamma \mathrm{R}_{\mathrm{sp}} \mathrm{T}_{1}}}$ & 0.37 & 0.37 \\
\hline $\mathrm{Re}_{\mathrm{u}}=\frac{\rho_{\mathrm{cg}} \mathrm{u}_{\mathrm{cg}} \mathrm{R}}{\eta_{\mathrm{cg}}}$ & $0.8 * 10^{6}$ & $0.8 * 10^{6}$ \\
\hline $\mathrm{cw}=\frac{\rho_{c g} \mathrm{u}_{\mathrm{cg}} \mathrm{A}_{\mathrm{cg}}}{\eta_{\mathrm{cg}} \mathrm{R}}$ & 1000 & 2000 \\
\hline
\end{tabular}

\section{RESULTS AND DISCUSSION}

In the following, the numerical results of the flow field in the one-stage axial flow turbine for the two cases with different cooling gas mass flow rates are discussed. The operating conditions are listed in Table 1. The data for CW2K is taken from (Pogorelov et al. 2019). For both operating conditions, it takes approximately 40 full rotor rotations until the flow field is fully developed. This requires about 2100 hours of computing time on 4800 cores on a CRAY XC40. A snapshot of the flow variables requires approximately $58.5 \mathrm{~Gb}$ of disc space. For the analysis only the final 3 rotations are considered. First, the mean flow fields in both LES are compared. Afterwards, the instantaneous flow field is analysed. Finally, the numerical results are compared against experimental data.

Figure 4 shows the azimuthal distribution of the time averaged effective radial velocity inside the rim seal gap at $\mathrm{r} / \mathrm{R}=0.98$ (Figure 3) for CW1K (solid) and CW2K (dashed). The effective radial velocity is the radial velocity spatially averaged in the axial direction. In both LES the effective radial velocity evidences 30 distinct minima and maxima along the circumference, which are associated with the nonaxisymmetric pressure distribution caused by the 30 stator blade wakes. The effective radial velocity becomes negative in the stator blade wake regions, which, therefore, is externally-induced ingress. In $\mathrm{CW} 1 \mathrm{~K}$ the averaged radial velocity is slightly lower due to reduced cooling gas mass flux.

The root-mean-square (rms) values of the effective radial velocity fluctuation inside the rim seal gap are shown in Figure 5. The reduction of the cooling gas mass flux leads to reduced fluctuations in the rim seal gap.

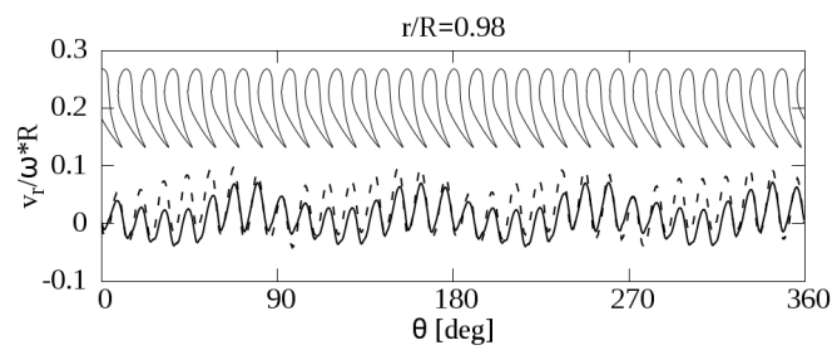

Figure 4 Azimuthal distribution of the time averaged effective radial velocity; CW1K (solid), CW2K (dashed); $r / R=0.98$ 


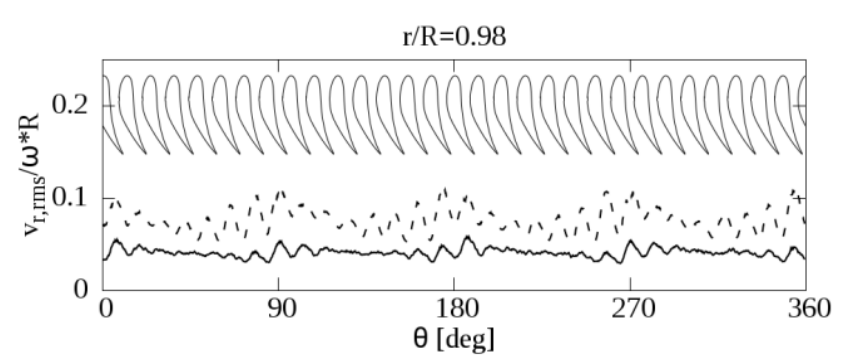

Figure 5 Azimuthal distribution of the effective radial velocity fluctuations; CW1K (solid), CW2K (dashed); $r / R=0.98$

Figure 6 compares the radial distributions of the time averaged velocity components and pressure inside the wheel space at the fixed azimuthal position $\Theta=0^{\circ}$. The distributions in two axial positions are investigated, i.e., near the stator wall at $\mathrm{x} / \mathrm{s}_{\mathrm{c}}=-8.7$ and near the rotor wall at $\mathrm{x} / \mathrm{s}_{\mathrm{c}}=0.5$ (Figure 3). Due to the lower mass flux in CW1K (solid), the cooling gas undergoes a stronger acceleration in the circumferential direction than in CW2K (dashed). The distributions of the radial velocities indicate the disk pumping effect, where the cooling gas is radially pumped outward near the rotor wall and flows radially inward in the stator boundary layer, which generates a large vortex structure in the time averaged flow field. The exact position of this vortex seems to depend on the cooling gas mass flux. The time averaged pressure level inside the wheel space is similar for both cooling gas mass fluxes. The pressure is normalized by the time and azimuthally averaged pressure inside the rim seal gap $p_{\text {gap }}$ at $\mathrm{r} / \mathrm{R}=0.98$. The deviation between both cases is less than $1 \%$.

Figure 7 shows the radial distributions of the rms values in the same axial and azimuthal positions. The rms values for the azimuthal velocity in CW1K (solid) are lower than those in $\mathrm{CW} 2 \mathrm{~K}$ (dashed), while this behavior is reversed for the radial velocity. The pressure fluctuations show the strongest deviation. In $\mathrm{CW} 2 \mathrm{~K}$, the pressure rms values are almost constant. In CW1K, they fluctuate significantly about a mean value which exceeds the rms values in $\mathrm{CW} 2 \mathrm{~K}$ by a factor two.

To identify the reason for the increased pressure fluctuations, the instantaneous flow field inside the wheel space is investigated more thoroughly. The pressure signals inside the wheel space have been recorded over 3 full rotor rotations and sampled in 408 radially distributed bins along the stator wall. For these signals cross-correlations are computed relative to the signal at location $r / R=0.96$. The results are depicted in Figure 8 . The square pattern for CW1K (left) indicates the existence of a standing wave inside the rotor-stator wheel space. For CW2K (right), no distinct pattern can be observed.
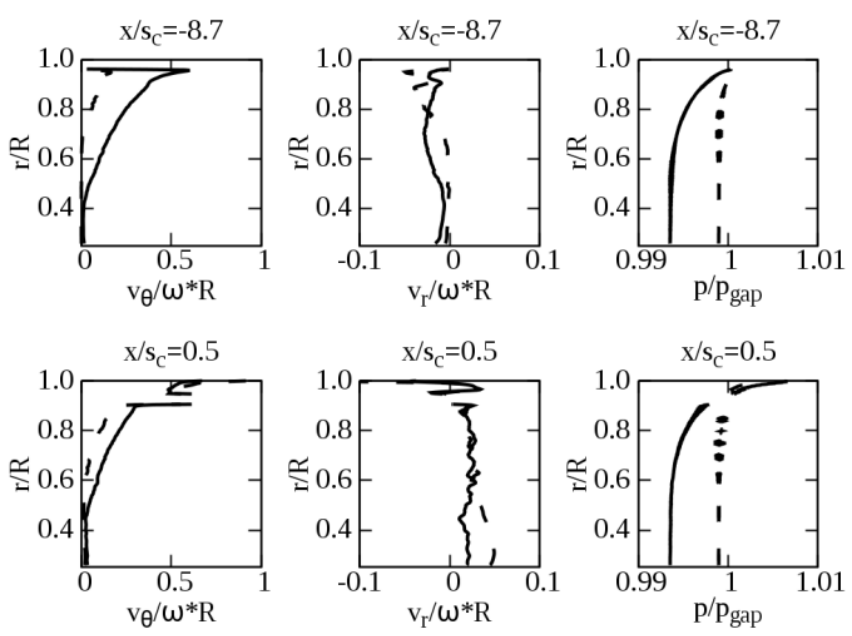

Figure 6 Time averaged radial distributions of the velocity components and the pressure at two axial positions; CW1K (solid), CW2K (dashed)
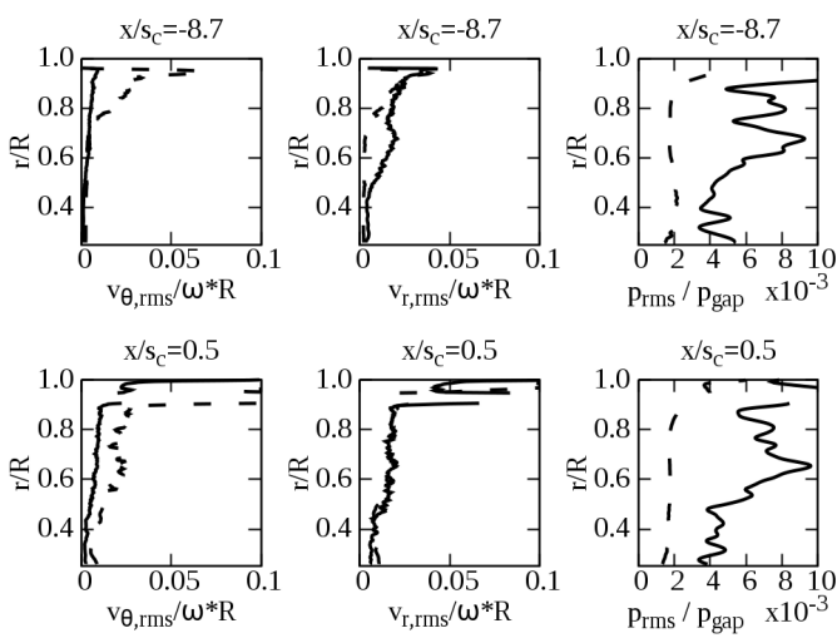

Figure 7 Radial distributions of the velocity components and the pressure fluctuations at two axial positions; CW1K (solid), CW2K (dashed)
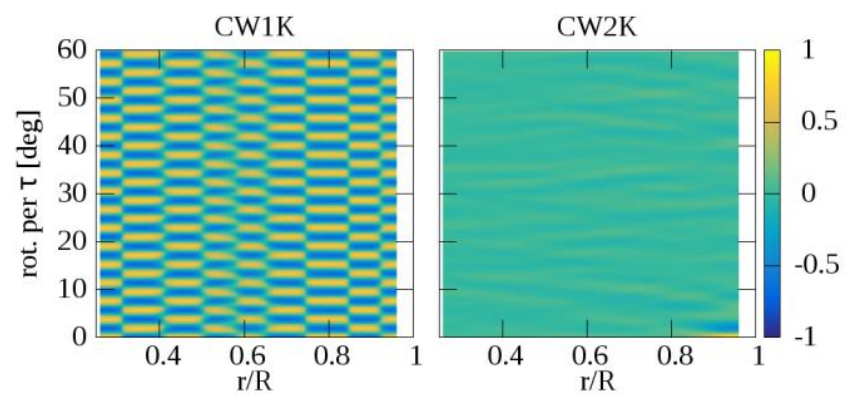

Figure 8 Cross-correlations computed from the pressure fluctuations along the stator wall inside the wheel space; CW1K (left), CW2K (right) 
This is further confirmed when the energy spectra computed form these cross-correlations are investigated. Figure 9 shows that the energy spectrum for CW1K (top) features a distinct peak at a frequency of approximately 94.33 times the rotor speed $n$, which exists along the entire stator wall. For the sake of conciseness only the spectrum at the radial position $\mathrm{r} / \mathrm{R}=0.6$ is shown. The energy spectrum of CW2K (bottom) only shows a minor peak at the blade passage frequency (BPF) $\mathrm{f} / \mathrm{n}=62$. Note that the low local minima and maxima in the pressure rms values of CW1K in Figure 7 coincide with the nodes of the standing wave seen in the respective cross-correlation in Figure 8.

This dominant frequency in $\mathrm{CW} 1 \mathrm{~K}$ is also found in the instantaneous flow field inside the rim seal gap at $\mathrm{r} / \mathrm{R}=$ 0.98. The signals of the pressure and the effective radial velocity were recorded over 3 full rotor rotations along the entire circumference in 1440 equidistantly spaced positions. Using the same procedure as described for the energy spectra in Figure 9, the energy spectra of the pressure fluctuations and the fluctuations of the effective radial velocity inside the rim seal gap are obtained. Due to the page limit the energy spectra are only shown for the position $\Theta=180^{\circ}$. The pressure energy spectrum of CW2K (bottom) in Figure 10 only evidences the blade passage frequency at $\mathrm{f} / \mathrm{n}=62$. For $\mathrm{CW} 1 \mathrm{~K}$ (top), however, not only the blade passage frequency but a wide range of frequencies from $\mathrm{f} / \mathrm{n}=30$ to $\mathrm{f} / \mathrm{n}=300$ is apparent. The most dominant frequency is the one which has already been determined inside the wheel space at $\mathrm{f} / \mathrm{n} \approx 94.33$.

The energy spectrum of the effective radial velocity fluctuations for CW1K in Figure 11 (top) shows the same frequency as the pressure fluctuations for $\mathrm{CW} 1 \mathrm{~K}$ in Figure 10 , however, with differently pronounced amplitudes. Below the dominant frequency $\mathrm{f} / \mathrm{n} \approx 94.33$, only a band of elevated frequencies is observed. This is also apparent for $\mathrm{CW} 2 \mathrm{~K}$ (bottom).
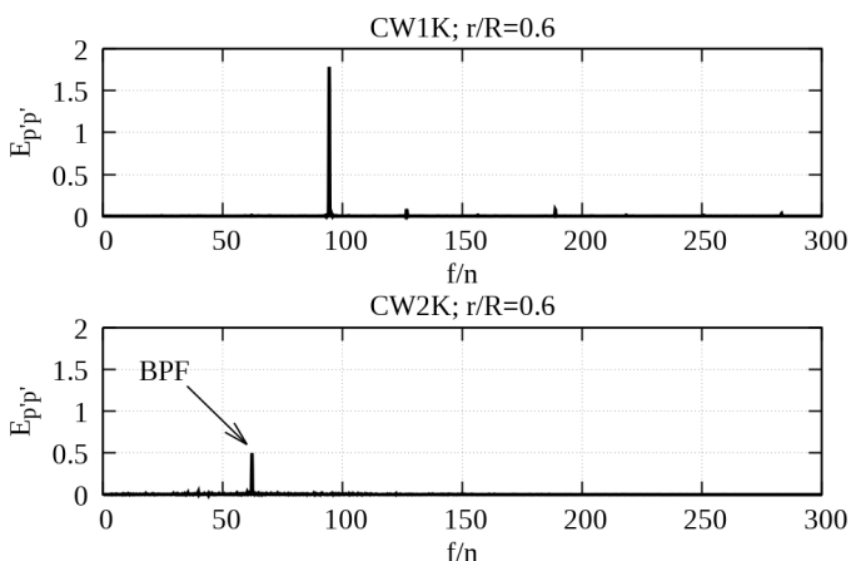

Figure 9 Energy spectra computed from crosscorrelations of the pressure fluctuations along the stator wall inside the wheel space; CW1K (top), CW2K (bottom); $x / s_{c}=-8.7$
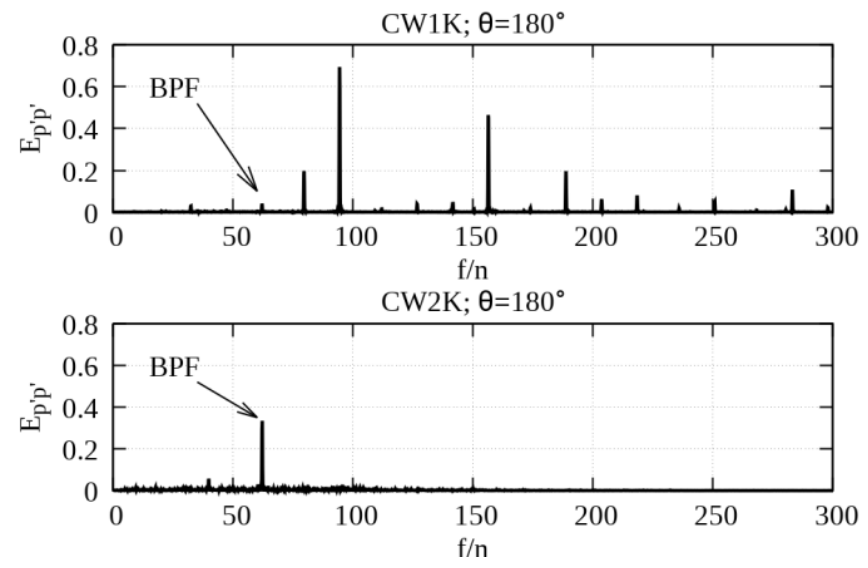

Figure 10 Energy spectra computed from crosscorrelations of the pressure fluctuations inside the rim seal gap; CW1K (top), CW2K (bottom); r/R=0.98
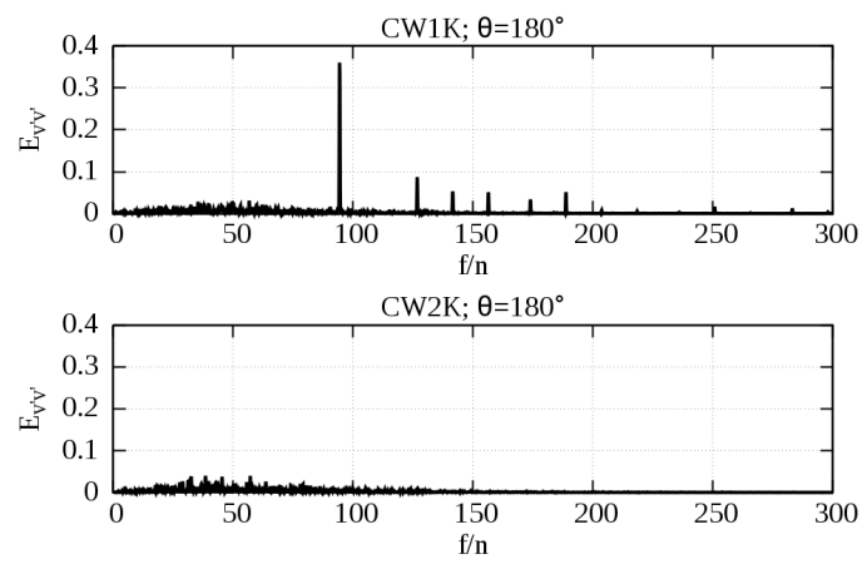

\section{Figure 11 Energy spectra computed from cross- correlations of the effective radial velocity fluctuations inside the rim seal gap; CW1K (top), CW2K (bottom); r/R=0.98}

The acoustic eigenfrequencies of a closed pipe can be calculated by

$$
\mathrm{f}=\frac{\mathrm{m}}{2 \cdot \mathrm{L}} \cdot \mathrm{a},
$$

with a the speed of sound, L the length of the resonator, and $m$ the order of the harmonic. Inside the wheel space the Mach number is low and the speed of sound almost constant. For $\mathrm{CW} 1 \mathrm{~K}$, the ratio of the speed of sound and the circumferential speed is approximately $\mathrm{a} / \omega \mathrm{R}=2.853$. Using the height of the wheel space $\mathrm{L}=\mathrm{R}-\left(2 \cdot \mathrm{d}+\mathrm{s}_{\mathrm{c}}\right)-$ $r_{h}=86.65 \mathrm{~mm}$ the harmonics can be determined. The data are listed in Table 2. Of course, to approximate the wheel space by a closed pipe is pretty crude, since the mean pressure increases radially, and the mean flow velocity is not zero. Nevertheless, the $8^{\text {th }}$ harmonic lies very close to $f / n \approx$ 94.33.

The comparison of the LES data for CW1K from Figure 10 with the theoretical finding shows a good match. The strongest deviations between the theoretical values and the 
Table 2 Harmonics of a closed pipe normalized by rotor speed $\mathbf{f} / \mathbf{n}$

\begin{tabular}{|l|l|l|l|l|l|}
\hline $\mathrm{m}$ & 7 & 8 & 11 & 12 & 13 \\
\hline Theory & 84.17 & 96.20 & 132.27 & 144.29 & 156.32 \\
\hline LES & 79.53 & 94.33 & 126.6 & 141.5 & 156.3 \\
\hline rel. Error & $-5.5 \%$ & $-1.9 \%$ & $4.2 \%$ & $-1.9 \%$ & $-0.01 \%$ \\
\hline $\mathrm{m}$ & 16 & 17 & 18 & 21 & 24 \\
\hline Theory & 192.39 & 204.42 & 216.44 & 252.51 & 288.59 \\
\hline LES & 188.7 & 203.5 & 218.3 & 250.6 & 283.1 \\
\hline rel. Error & $-1.9 \%$ & $-0.5 \%$ & $0.9 \%$ & $-0.8 \%$ & $-1.9 \%$ \\
\hline
\end{tabular}

LES occur for the frequencies being close to a multiple of the blade passage frequency, i.e.

$$
\frac{f}{n}=\mathrm{x} * 62 \text {. }
$$

Apparently, the rotor-stator interaction and the turbulent fluctuations inside the shear layer between the main flow and the exiting cooling gas excite several of the wheel spaces' harmonics. This, in turn, results in the development of a standing wave inside the wheel space.

To analyze the impact of the dominant $8^{\text {th }}$ harmonic at $\mathrm{f} / \mathrm{n} \approx 94.33$ a fourth-order Butterworth bandpass filter is applied to the flow field of the effective radial velocity inside the rim seal gap. The result is shown in Figure 12 for a time period of $60^{\circ}$ rotor rotation. A square pattern consisting of 30 fields in the azimuthal direction and a strong fluctuation in time is evident. The 30 azimuthal fields correspond to the 30 stator blades. The temporal fluctuation repeats approximately every $3.82^{\circ}$ rotor rotation and corresponds to the standing wave inside the wheel space. Interestingly, this temporal fluctuation leads to alternating positive and negative values of the effective radial velocity with an amplitude of the same order as the mean effective radial velocity inside the rim seal gap (Figure 4). Hence, the standing wave triggers alternating cold gas egress and hot gas ingress. Therefore, it has a major impact on the cooling effectiveness.

The impact of the $8^{\text {th }}$ harmonic on the cooling effectiveness is displayed by the radial distribution of the time and circumferentially averaged cooling effectiveness for CW1K and CW2K in Figure 13. The cooling effectiveness is computed by

$$
\eta=\frac{Y(r)-Y_{h g}}{Y_{c g}-Y_{h g}}
$$

where $\mathrm{Y}$ is the concentration of a tracer gas mixed into the cooling gas. The concentration is obtained from a passive scalar transport equation. The subscripts hg and cg denote the concentrations at the main flow and the cooling gas inlet. In both cases the LES results match the experimental data from (Bohn and Wolff 2001) convincingly. For CW1K, the cooling effectiveness is significantly reduced compared to CW2K, since none of the wheel space's harmonics are excited for CW2K.

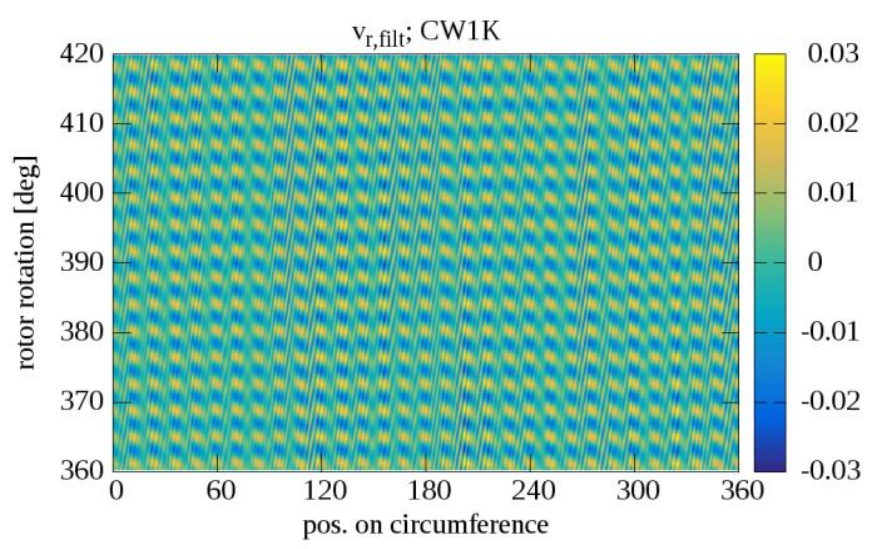

Figure 12 Filtered effective radial velocity inside the rim seal gap at $r / R=0.98$; CW1K

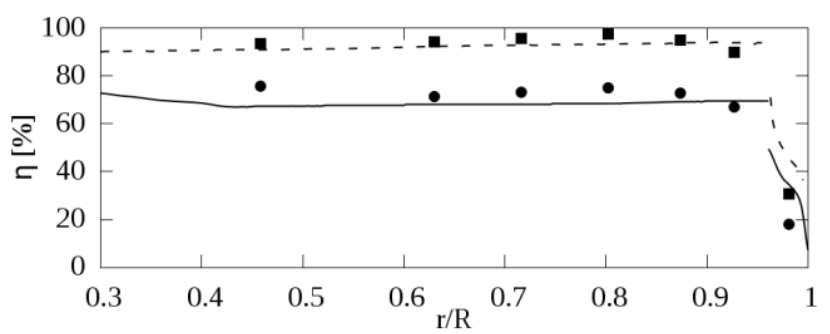

Figure 13 Radial distribution of the time and azimuthally averaged cooling effectiveness; LES: CW1K (solid line), CW2K (dashed line), exp. data (Bohn and Wolff 2001): CW1K (full circles), CW2K (full squares)

\section{CONCLUSIONS}

The flow field for two cooling gas mass flow rates of a one-stage axial flow turbine has been investigated. Approximately 40 full rotor rotations were necessary to reach a statistically converged flow field.

It was shown that the reduction of the cooling gas mass flux has only little effect on the time averaged flow field inside the wheel space. The instantaneous flow field, however, is strongly influenced by the amount of cooling gas. For the lower cooling gas mass flux $(\mathrm{CW} 1 \mathrm{~K})$, several of the wheel space's harmonics are excited. This leads to the development of a standing wave inside rotor-stator wheel space, which has a significant impact on the radial velocity inside the rim seal gap. Over one time period of that wave, cooling gas is blown out of the wheel space, followed by a suction of hot gas from the main annulus into the wheel space. For the higher cooling gas mass flux (CW2K), the wheel space's harmonics are not excited.

The suction of hot gas into the wheel space in $\mathrm{CW} 1 \mathrm{~K}$, caused by the excited $8^{\text {th }}$ harmonic of the wheel space, results in a significantly reduced cooling effectiveness compared to CW2K. In brief, it can be concluded that there is a critical cooling gas mass flux above which the turbulent fluctuations inside the rotor-stator cavity are stabilized. 


\section{ACKNOWLEDGMENTS}

The authors gratefully acknowledge the Gauss Centre for Supercomputing e.V. (www.gauss-centre.eu) for funding this project by providing computing time on the GCS Supercomputer HAWK at Höchstleistungsrechenzentrum Stuttgart (www.hlrs.de).

This study was funded under Grant No. $19198 \mathrm{~N}$ by the German Federal Ministry of Economics and Technology via the Arbeitsgemeinschaft industrieller Forschungsvereinigungen Otto von Guericke e.V. (AiF), Forschungsvereinigung Verbrennungskraftmaschinen e.V.

\section{References}

Batten, P., Goldberg, U. and Chakravarthy, S. (2004), 'Interfacing Statistical Turbulence Closures with Large-Eddy Simulation', AIAA Journal, 42/3: 485-492.

Beard, P. F., Gao, F. and Chana, K. S. (2016), 'Unsteady Flow Phenomena in Turbine Rim Seals', Journal of Engineering for Gas Turbines and Power, 139/3.

Berger, M. and Aftosmis, M. (2012), 'Progress Towards a Cartesian Cut-Cell Method for Viscous Compressible Flow', AIAA 2012-1301.

Bohn, D. and Wolff, M. (2001), Entwicklung von Berechnungsansätzen zur Optimierung von Sperrgassystemen für Rotor/Stator-Kavitäten in Gasturbinen (Frankfurt am Main), FVV-Vorhaben Nr:067270.

Bohn, D. and Wolff, M. (2003), 'Improved Formulation to Determine Minimum Sealing Flow - $\mathrm{Cw}$, min - for Different Sealing Configurations', ASME Paper no. GT2003-38465.

Bohn, D., Decker, A., Ma, H. and Wolff, M. (2003), 'Influence of Sealing Air Mass Flow on the Velocity Distribution In and Inside the Rim Seal of the Upstream Cavity of a 1.5-Stage Turbine', ASME Paper no. GT200338459.

Boris, J. P., Grinstein, F. F., Oran, E. S. and Kolbe, R. L. (1992), 'New insights into large eddy simulation', Fluid Dynamics Research., 10/4-6: 199-228.

Cao, C., Chew, J. W., Millington, P. R. and Hogg, S. I. (2004), 'Interaction of Rim Seal and Annulus Flows in an Axial Flow Turbine', Journal of Engineering for Gas Turbines and Power, 126/4: 786-793.

Chew, J. W., Gao, F., and Palermo, D. M. (2019), 'Flow mechanisms in axial turbine rim sealing', Proceedings of the Institution of Mechanical Engineers, Part C: Journal of Mechanical Engineering Science, 233/23-24: 7637-7657.

Chilla, M., Hodson, H. and Newman, D. (2013), 'Unsteady Interaction Between Annulus and Turbine Rim Seal Flows', Journal of Turbomachinery, 135/5.

Jakoby, R., Zierer, T., Lindblad, K., Larsson, J., DeVito, L., Bohn, D., Funcke, J. and Decker, A. (2004), 'Numerical Simulation of the Unsteady Flow Field in an Axial Gas Turbine Rim Seal Configuration', ASME Paper no. GT200453829.

Johnson, B. V., Mack, G. J., Paolillo, R. E. and Daniels, W. A. (1994), 'Turbine rim seal gas path flow ingestion mechanisms', in , 30th Joint Propulsion Conference and
Exhibit (Reston, Virigina: American Institute of Aeronautics and Astronautics).

Laskowski, G. M., Bunker, R. S., Bailey, J. C., Ledezma, G., Kapetanovic, S., Itzel, G. M., Sullivan, M. A. and Farrell, T. R. (2009), 'An Investigation of Turbine Wheelspace Cooling Flow Interactions With a Transonic Hot Gas Path-Part 2: CFD Simulations', Journal of Turbomachinery, 133/4.

Lintermann, A., Meinke, M., and Schröder, W. (2020), 'Zonal Flow Solver (ZFS): a highly efficient multi-physics simulation framework', International Journal of Computational Fluid Dynamics, 2020: 1-28.

Lintermann, A., Schlimpert, S., Grimmen, J. H., Günther, C., Meinke, M. and Schröder, W. et al. (2014), 'Massively parallel grid generation on HPC systems', Computer Methods in Applied Mechanics and Engineering, 277: 131-153.

Meinke, M., Schröder, W., Krause, E. and Rister, T. (2002), 'A comparison of second- and sixth-order methods for large-eddy simulations', Computers \& Fluids, 31/4-7: 695-718.

O’Mahoney, T. S. D., Hills, N. J., Chew, J. W. and Scanlon, S. (2010), 'Large-Eddy Simulation of Rim Seal Ingestion', ASME Paper no GT2010-22962.

Pogorelov, A., Meinke, M., and Schröder, W. (2019), 'Large-Eddy Simulation of the Unsteady Full 3D Rim Seal Flow in a One-Stage Axial-Flow Turbine', Flow Turbulence Combust, 102/1: 189-220.

Pogorelov, A., Schneiders, L., Meinke, M. and Schröder, W. (2018), 'An Adaptive Cartesian Mesh Based Method to Simulate Turbulent Flows of Multiple Rotating Surfaces', Flow Turbulence Combust, 100/1: 19-38.

Rabs, M., Benra, F.-K., Dohmen, H. J. and Schneider, O. (2009), 'Investigation of Flow Instabilities Near the Rim Cavity of a 1.5 Stage Gas Turbine', ASME Paper no. GT2009-59965.

Rudzinski, B. (2009), Experimentelle Untersuchung des Heißgaseinzuges in die Rotor-Stator-Zwischenräume einer eineinhalbstufigen Turbine für unterschiedliche Dichtkonfigurationen, Zugl.: Aachen, Techn. Hochsch., Diss., 2009 (Energietechnik; 1. Aufl., München: Verl. Dr. Hut).

Schädler, R., Kalfas, A. I., Abhari, R. S., Schmid, G. and Voelker, S. (2017), 'Modulation and Radial Migration of Turbine Hub Cavity Modes by the Rim Seal Purge Flow', Journal of Turbomachinery, 139/1.

Schneiders, L., Günther, C., Meinke, M. and Schröder, W. (2016), 'An efficient conservative cut-cell method for rigid bodies interacting with viscous compressible flows', Journal of Computational Physics, 311: 62-86.

Schuepbach, P., Abhari, R. S., Rose, M. G., Germain, T., Raab, I. and Gier, J. (2010), 'Effects of Suction and Injection Purge-Flow on the Secondary Flow Structures of a HighWork Turbine', Journal of Turbomachinery, 132/2.

Sutherland, W. (1893), 'LII. The viscosity of gases and molecular force', The London, Edinburgh, and Dublin Philosophical Magazine and Journal of Science, 36/223: 507-531. 